\title{
EQUIVARIANT MAPS FOR HOMOLOGY REPRESENTATIONS
}

\author{
RONALD M. DOTZEL
}

(Communicated by Frederick R. Cohen)

\begin{abstract}
If $Y$ is a homotopy representation of the finite group $G$ of order $n$ and $X$ is a finite $G$-CW complex such that, for each subgroup $H$ of $G$, $H_{*}\left(X^{H} ; \mathbb{Z}_{n}\right)=H_{*}\left(Y^{H} ; \mathbb{Z}_{n}\right)$ then there exists a $G$-map $f: X \rightarrow Y$ such that $f_{*}^{H}: H_{*}\left(X^{H} ; \mathbb{Z}_{n}\right) \rightarrow H_{*}\left(Y^{H} ; \mathbb{Z}_{n}\right)$ is an isomorphism for each subgroup $H$.
\end{abstract}

\section{INTRODUCTION}

Let $G$ be a finite group of order $n$. A homotopy representation [tD4] of $G$ is a finite $G$-CW complex $Y$ such that, for each $H \leq G, Y^{H}$ is an $n(H)$ dimensional complex, homotopy equivalent to a sphere $S^{n(H)}$ for some integer $n(H) \geq-1$ (-1 signifies that $Y^{H}$ is empty). Natural examples of homotopy representations are the unit spheres of orthogonal representations. From the viewpoint of the function defined on subgroups of $G$ by the numbers $n(H)$ (the dimension function), if $G$ is a $p$-group these are the only examples [DH, tD4]. For general $G$, homotopy representations can deviate greatly from the linear situation, either in terms of dimension functions or linking of fixed point sets (even $p$-groups have homotopy representations with nonstandard linking of fixed sets) [tD3].

Suppose that $X$ is a finite $G$-CW complex such that, for each $H \leq G$, $X^{H}$ has the $\mathbb{Z}_{n}$-homology of a sphere (where $G$ has order $n$ ). Such an $X$ is called a $\mathbb{Z}_{n}$-homology representation of $G$ (compare [tD4]). If $X$ and $Y$ are $\mathbb{Z}_{n}$-homology representations of $G$ such that, for each $H \leq G, X^{H}$ and $Y^{H}$ have the same $\mathbb{Z}_{n}$-homology then $X$ and $Y$ are $\mathbb{Z}_{n}$-homology equivalent. Suppose now that $X$ is a $\mathbb{Z}_{n}$-homology representation of $G$ which is homology equivalent to a homotopy representation $Y$ of $G$. In this situation we obtain

Theorem. There exists a G-map $\phi: X \rightarrow Y$ such that, for any $H \leq G$,

$$
\phi_{*}^{H}: H_{*}\left(X^{H} ; \mathbb{Z}_{n}\right) \rightarrow H_{*}\left(Y^{H} ; \mathbb{Z}_{n}\right)
$$

is an isomorphism.

In particular, let $G$ be a $p$-group. If $X$ is a $\mathbb{Z}_{p}$-homology representation of $G$ then by [DH, tD4] there exists an orthogonal representation $V$ of $G$ such that $X$ is homology equivalent to $S(V)$.

Received by the editors May 7, 1991 and, in revised form, October 19, 1992.

1991 Mathematics Subject Classification. Primary 57S30. 
Corollary. Let $G$ be a p-group and $X$ a finite $G-C W$ complex. If $X$ is a $\mathbb{Z}_{p^{-}}$ homology sphere then there is a G-map $\phi: X \rightarrow S(V)$ such that $\phi_{*}^{H}: H_{*}\left(X^{H} ; \mathbb{Z}_{p}\right)$ $\rightarrow H_{*}\left(S(V)^{H} ; \mathbb{Z}_{p}\right)$ is an isomorphism for all $H \leq G$.

This corollary improves on [DH], where the existence of a stable map was shown.

In this section we will prove the theorem, modulo several auxiliary results which are proved in $\S 2$. If $H \leq G$ with normaliser $N H$, as is standard, we denote $\mathrm{NH} / \mathrm{H}$ by $\mathrm{WH}$.

Let $X$ be as in the statement of the theorem. Suppose $\left(H_{1}\right), \ldots,\left(H_{m}\right)$ are the conjugacy classes of isotropy subgroups of $G$ ordered in such a way that $\left(H_{i}\right)<\left(H_{j}\right)$ (i.e., $H_{i}$ subconjugate to $\left.H_{j}\right)$ implies $j<i$. Put $X_{j}=$ $\left\{x \in X \mid\left(G_{x}\right)=\left(H_{i}\right)\right.$ for some $\left.i \leq j\right\}$. Each $X_{j}$ is a $G$ subspace of $X$ and $X_{1} \subseteq X_{2} \subseteq \cdots \subseteq X_{m}=X$. We would like to construct a $G$-map $\phi_{m}: X \rightarrow Y$ satisfying the condition of the theorem by induction over the $X_{i}$. Let $X_{0}=\varnothing$, and suppose we have found $\phi_{i}: X_{i} \rightarrow Y$ such that whenever $(H)=\left(H_{j}\right)$, for some $j \leq i,\left(\phi_{i}^{H}\right)_{*}: H_{*}\left(X_{i}^{H} ; \mathbb{Z}_{n}\right) \rightarrow H_{*}\left(Y^{H} ; \mathbb{Z}_{n}\right)$ is an isomorphism. We would like to extend $\phi_{i}$ to a $G$-map $\phi_{i+1}: X_{i+1} \rightarrow$ $Y$. By [tD2, 8.1.5] such extensions are in bijection with the $W H$ extensions $\phi_{i+1}^{H}: X_{i+1}^{H} \rightarrow Y^{H}$ of $\phi_{i}^{H}: X_{i}^{H} \rightarrow Y^{H}$ for all $H$ such that $(H)=\left(H_{i+1}\right)$. Let $H=H_{i+1}$. We look for such an extension $\phi_{i+1}^{H}: X_{i+1}^{H}=X^{H} \rightarrow Y^{H}$ which induces a homology isomorphism. If $n(H)=0$ then $X^{H}$ has two acyclic components and the definition of $\phi_{i+1}^{H}$ is clear. If $n(H) \geq 1$, since $Y^{H}$ is $(n(H)-1)$-connected, certainly there are equivariant extensions of $\phi_{i}^{H}$ to the $n(H)$-skeleton, $\phi_{i+1}^{H}:\left(X^{H}\right)^{(n(H))} \cup X_{i}^{H} \rightarrow Y^{H} . \phi_{i}^{M}: X^{M} \rightarrow Y^{M}$ induces a $\mathbb{Z}_{n^{-}}$ homology isomorphism whenever $H<M$. It follows using Mayer-Vietoris and Smith theory that there is an extension of $\phi_{i}^{H}$ to the $n(H)$-skeleton which induces a $\mathbb{Z}_{n}$-homology epimorphism. Obstructions to extending $\phi_{i+1}^{H}$ to the $(k+1)$-skeleton $(k \geq n(H))$ lie in the group $H_{W H}^{k+1}\left(X^{H}, X_{i}^{H} ; \tilde{\omega}_{k}\left(Y^{H}\right)\right)$ (see [B]). Because $W H$ acts freely off $X_{i}^{H}$, this group is the $(k+1)$-cohomology of the cochain complex $\operatorname{Hom}_{\mathbb{Z}[W H]}\left(C_{*}\left(X^{H}, X_{i}^{H} ; \mathbb{Z}\right), \pi_{k}\left(Y^{H}\right)\right)=C^{*}$. By Proposition 2 this cohomology group is torsion-prime to $n=|G|$. By Proposition 3 if $\theta$ is an obstruction, say of order $d$, then there is a $W H$ map $f: Y^{H} \rightarrow Y^{H}$ of degree of power of $d$ such that $f \circ \phi_{i+1}^{H}$ can be extended to the $(k+1)$-skeleton. Eventually we arrive at a $W H$ map $\phi_{i+1}^{H}: X_{i+1}^{H}=X^{H} \rightarrow Y^{H}$, which by construction induces a $\mathbb{Z}_{n}$-homology isomorphism. Finally $\phi_{i+1}^{H}$ yields a $G$-map $\phi_{i+1}: X_{i+1} \rightarrow Y$ by defining $\phi_{i+1}(x)=g \phi_{i+1}^{H_{i+1}}\left(g^{-1} x\right)$ if $x \in g X^{H_{i+1}}=X^{g H_{i+1} g^{-1}}$ [tD2, 8.1.5].

In this section we prove the several results used in $\S 1$. We begin with a lemma needed later to establish Proposition 2.

Lemma 1. Let $X$ be a $\mathbb{Z}_{n}$-homology representation of the finite group $G$ of order $n$ which is homology equivalent to the homotopy representation $Y$. Suppose that 
$H \leq G$ and $\Omega$ is a collection of subgroups of $G$ which contain $H$. Then

$$
H_{*}\left(X^{H}, \bigcup_{\Omega} X^{M} ; \mathbb{Z}_{n}\right)=0, \quad *>n(H) .
$$

Proof. Use induction on $n(H)$ and $|\Omega|$. So suppose $M_{0} \in \Omega$ has $n\left(M_{0}\right)<$ $n(H)$. Set $\Omega_{0}=\Omega-\left\{M_{0}\right\}$. Throughout we use $\mathbb{Z}_{n}$-coefficients. By MayerVietoris,

$$
\begin{aligned}
& H_{1}\left(X^{H}, X^{M_{0}}\right) \oplus H_{1}\left(X^{H}, \bigcup_{\Omega_{0}} X^{M}\right) \\
& \quad \rightarrow H_{i}\left(X^{H}, \bigcup_{\Omega} X^{M}\right) \rightarrow H_{i-1}\left(X^{H}, \bigcup_{\Omega_{0}} X^{M M_{0}}\right) .
\end{aligned}
$$

It follows immediately by induction that $H_{i}\left(X^{H}, \bigcup_{\Omega} X^{M} ; \mathbb{Z}_{n}\right)$ is zero for $i>$ $n(H)+1$. For $i=n(H)+1$, by induction we have

$$
0 \rightarrow H_{n(H)+1}\left(X^{H}, \bigcup_{\Omega} X^{M}\right) \rightarrow H_{n(H)}\left(X^{H}, \bigcup_{\Omega} X^{M M_{0}}\right)
$$

$$
\rightarrow H_{n(H)}\left(\left(X^{H}, X^{M_{0}}\right) \oplus H_{n(H)}\left(X^{H}, \bigcup_{\Omega_{0}} X^{M}\right) .\right.
$$

Since $n\left(M_{0}\right)<n(H)$, by induction again $H_{n(H)}\left(X^{M_{0}}, \bigcup_{\Omega_{0}} X^{M M_{0}}\right)=0$. So the inclusion $H_{n(H)}\left(X^{H}, \bigcup_{\Omega_{0}} X^{M M_{0}}\right) \rightarrow H_{n(H)}\left(X^{H}, X^{M_{0}}\right)$ is a monomorphism as is seen by looking at the triple $\left(X^{H}, X^{M_{0}}, \bigcup_{\Omega_{0}} X^{M M_{0}}\right)$. Therefore, the rightmost map in $(* *)$ is a monomorphism, so $H_{n(H)+1}\left(X^{H}, \bigcup_{\Omega} X^{M}\right)=0$.

On the other hand if every $M \in \Omega$ has $n(M)=n(H)$, let $M_{0} \in \Omega$ be arbitrary. If $M_{1}, M_{2} \in \Omega$ then $n\left(M_{1}\right)=n\left(M_{2}\right)=n(H)$. Now $X$ is homology equivalent to the homotopy representation $Y$. Apply relative Mayer-Vietoris to the pairs $\left(Y^{H}, Y^{M_{1}}\right)$ and $\left(Y^{H}, Y^{M_{2}}\right)$. One has

$$
0 \rightarrow H_{n(H)+1}\left(Y^{H}, Y^{M_{1}} \cup Y^{M_{2}}\right) \rightarrow H_{n(H)}\left(Y^{H}, Y^{M_{1} M_{2}}\right) \rightarrow 0 .
$$

Since $Y^{H}$ has no cells in dimensions higher than $n(H)$,

$$
H_{n(H)+1}\left(Y^{H}, Y^{M_{1}} \cup Y^{M_{2}}\right)=0 .
$$

This shows that $n\left(M_{1} M_{2}\right)=n(H)$, where $M_{1} M_{2}$ is the subgroup generated by $M_{1}, M_{2}$. Now from (*) (with $M_{0}$ arbitrary such that $n\left(M_{0}\right)=n(H)$ ) it follows by induction on $|\Omega|$ that $H_{i}\left(X^{H}, \bigcup_{\Omega} X^{M}\right)=0$ for $i>n(H)$.

Proposition 2. Suppose that $X$ is a $\mathbb{Z}_{n}$-homology representation of $G$ which is homology equivalent to a homotopy representation $Y$ of $G$, where $Y^{H} \simeq S^{n(H)}$ for all $H \leq G$. Let $k \geq n(H)$, and set

$$
C^{*}=\operatorname{Hom}_{\mathbb{Z}[W H]}\left(C_{*}\left(X^{H}, X_{i}^{H} ; \mathbb{Z}\right), \pi_{k}\left(Y^{H}\right)\right) .
$$

Then $H^{k+1}\left(C^{*}\right)$ is torsion-prime to $n=|G|$.

Proof. $C_{*}\left(X^{H}, X_{i}^{H} ; \mathbb{Z}\right)$ is a free $\mathbb{Z}[W H]$ chain complex. Let $p \mid n$. By [CE, pp. $\left.30,3^{\prime}\right]$ the cochain complex $\operatorname{Hom}_{\mathbb{Z}[W H]}\left(C_{*}\left(X^{H}, X_{i}^{H}, \mathbb{Z}\right), \mathbb{Z}_{p}\right)$ is chain isomorphic to $\operatorname{Hom}_{\mathbb{Z}_{p}[W H]}\left(C_{*}\left(X^{H}, X_{i}^{H} ; \mathbb{Z}_{p}\right), \mathbb{Z}_{p}\right)$. For the moment suppose that 
the cohomology of the latter cochain complex is zero in dimensions larger than $k$. For an abelian group $A$, let $A_{p}$ denote the subgroup of elements of order $p$. One has the two short exact sequences,

$$
\begin{gathered}
0 \rightarrow \pi_{k}\left(Y^{H}\right)_{p} \stackrel{j}{\rightarrow} \pi_{k}\left(Y^{H}\right) \rightarrow p \pi_{k}\left(Y^{H}\right) \rightarrow 0, \\
0 \rightarrow p \pi_{k}\left(Y^{H}\right) \stackrel{i}{\rightarrow} \pi_{k}\left(Y^{H}\right) \stackrel{\pi}{\rightarrow} \pi_{k}\left(Y^{H}\right) \otimes \mathbb{Z}_{p} \rightarrow 0 .
\end{gathered}
$$

These induce exact sequences in cohomology. To see that $H^{k+1}\left(C^{*}\right)$ is torsionprime to $p$, it suffices to know that the cochain complexes

$$
\operatorname{Hom}_{\mathbb{Z}[W H]}\left(C_{*}\left(X^{H}, X_{i}^{H}, \mathbb{Z}\right), \pi_{k}\left(Y^{H}\right)_{p}\right)
$$

and

$$
\operatorname{Hom}_{\mathbb{Z}[W H]}\left(C_{*}\left(X^{H}, X_{i}^{H}, \mathbb{Z}\right), \pi_{k}\left(Y^{H}\right) \otimes \mathbb{Z}_{p}\right)
$$

have zero cohomology in dimensions $k+2$ and $k+1$, respectively. But this follows by induction on the order of the groups $\pi_{k}\left(Y^{H}\right)_{p}, \pi_{k}\left(Y^{H}\right) \otimes \mathbb{Z}_{p}$ and the fact, now to be established, that $\operatorname{Hom}_{\mathbb{Z}_{p}[W H]}\left(C_{*}\left(X^{H}, X_{i}^{H} ; \mathbb{Z}_{p}\right), \mathbb{Z}_{p}\right)$ has zero cohomology in dimensions greater than $k$. Let $M$ be the cellular dimension of $X^{H}$. Then one has

$(* * *)$

$$
\begin{aligned}
& 0 \rightarrow C_{M}\left(X^{H}, X_{i}^{H} ; \mathbb{Z}_{p}\right) \stackrel{\partial_{M}}{\longrightarrow} \cdots \rightarrow C_{k+1}\left(X^{H}, X_{i}^{H} ; \mathbb{Z}_{p}\right) \\
& \stackrel{\partial_{k+1}}{\longrightarrow} C_{k}\left(X^{H}, X_{i}^{H} ; \mathbb{Z}_{p}\right) \rightarrow \frac{C_{k}\left(X^{H}, X_{i}^{H} ; \mathbb{Z}_{p}\right)}{\operatorname{Im} \partial_{k+1}} .
\end{aligned}
$$

This sequence is exact by Lemma 1 . For $j \geq k+1$ the $j$ th cohomology is $\operatorname{Ext}_{\mathbf{Z}_{p}[W H]}^{j-k}\left(\left(C_{k}\left(X^{H}, X_{i}^{H} ; \mathbb{Z}_{p}\right) / \operatorname{Im} \partial_{k+1}, \mathbb{Z}_{p}\right)\right.$. This is zero since the $\mathbb{Z}_{p}[W H]-$ module $\left(C_{k}\left(X^{H}, X_{i}^{H} ; \mathbb{Z}_{p}\right) / \operatorname{Im} \partial_{k+1}\right.$ is projective. For, by [R], projective over $\mathbb{Z}_{p}[W H]$ is the same as injective over $\mathbb{Z}_{p}[W H]$. So in $(* * *) C_{m}\left(X^{H}, X_{i}^{H} ; \mathbb{Z}_{p}\right)$ being projective is injective. Hence $\operatorname{Im} \partial_{M-1}=\operatorname{ker} \partial_{M-2}$ is projective, and hence injective. So $\operatorname{Im} \partial_{M-2}$ is projective and so on. Finally, the module $\left(C_{k}\left(X^{H}, X_{i}^{H} ; \mathbb{Z}_{p}\right)\right) / \operatorname{Im} \partial_{k+1}$ is projective.

Proposition 3. Suppose that $\theta \in H_{\mathbb{Z}[W H]}^{k+1}\left(X^{H}, X_{i}^{H} ; \tilde{\omega}_{k}\left(Y^{H}\right)\right)$ is an obstruction to extending the WH map $\phi_{i+1}^{H}: X_{i}^{H} \cup\left(X^{H}\right)^{(k)} \rightarrow Y^{H}$ to the $(k+1)$-skeleton of $X^{H}$. If $\theta$ has order $d$, there exists a WH map $f: Y^{H} \rightarrow Y^{H}$ of degree a power of $d$ such that $f \circ \phi_{i+1}^{H}$ can be extended.

Proof. Given an element $\alpha \in \pi_{k}\left(Y^{H}\right)=\pi_{k}\left(S^{n(H)}\right)$ of order $d$, one would like to multiply $\alpha$ by $d$ in following $\alpha$ by a map $f: Y^{H} \rightarrow Y^{H}$ of degree $d$. However, $f \circ \alpha$ might not be $d \alpha$ since composition is not left additive (but is right additive) [W, Chapter 11]. However, if $f: Y^{H} \rightarrow Y^{H}$ is a map of degree a suitable power $k$ of $d$ then $f \circ \alpha=k \alpha$. For by [W, Chapter 11, Theorem 8.9] if $n(H)$ is odd and $k \equiv 0$ or $1(\bmod 4)$ one has $(k) \circ \alpha=k \alpha$, where (k) denotes a map of degree $k$. If $n(H)$ is even and $k \equiv 0,+1$, or -1 $(\bmod 9)$ and $k \equiv 0$ or $1(\bmod 4)$ then $(k) \circ \alpha=k \alpha+\left(\begin{array}{l}k \\ 2\end{array}\right)(\alpha+(-i) \circ \alpha)$, where $i$ is the identity map on $S^{n(H)}$. Because composition is right additive, $d(\alpha+(-i) \circ \alpha)=0$. So if $d \mid\left(\begin{array}{l}k \\ 2\end{array}\right)$ then $(k) \circ \alpha=k \alpha$. One can check that $k=d^{6}$ works for all cases.

Finally according to [tD1, Theorem 4.11] one can find a $W H$ equivariant map $f: Y^{H} \rightarrow Y^{H}$ of degree $k$ which is the identity on $Y_{i}^{H}$ so long as $k \equiv 1$ 
$(\bmod |W H|)$. But since $d$ is relatively prime to $|W H|,\left(d^{6}\right)^{j} \equiv 1(\bmod \mid W H)$ for some $j$. So an $W H$ map of degree $d^{6 j}$ may be used.

\section{ACKNOWLEDGMENT}

The author thanks T. tom Dieck and S. Bauer for very useful conversations regarding this paper, and the referee for very careful comments. He also thanks the SFB at Göttingen for its hospitality and support.

\section{REFERENCES}

[B] G. Bredon, Equivariant cohomology theories, Lecture Notes in Math., vol. 34, SpringerVerlag, Berlin and New York, 1967.

[CE] H. Cartan and S. Eilenberg, Homological algebra, Princeton Math. Ser., vol. 19, Princeton Univ. Press, Princeton, NJ, 1956.

[tD1] T. tom Dieck, Transformation groups, de Gruyter, Berlin and New York, 1986.

[tD2] _ Transformation groups and representations theory, Lecture Notes in Math., vol. 766, Springer-Verlag, Berlin and New York, 1979.

[tD3] _ Geometric representation theory of compact lie groups, Proc. Internat. Congr. Math., Berkeley, 1986, pp. 615-622.

[tD4] _ Homotopiedarstellungen endlicher gruppen: dimensions-funktionen, Invent. Math. 67 (1982), 231-252.

[DH] R. Dotzel and G. Hamrick, p-Group actions on homology spheres, Invent. Math. 62 (1981), 437-442.

[R] D. S. Rim, Modules over finite groups, Ann. of Math. (2) 69 (1959), 700-712.

[W] G. Whitehead, Elements of homotopy theory, Graduate Texts in Math., vol. 61, SpringerVerlag, Berlin and New York, 1978.

Department of Mathematics, University of Missouri-St. Louis, St. Louis, Missouri 